\title{
Development of a Training System for Lathe Operation Using a Simulator
}

\author{
Tatsuya Yamaguchi ${ }^{1, *}$, Takashi Kawashimo ${ }^{1}$, Toshiyuki Matsumoto ${ }^{1}$, \\ and Daisuke Doyo ${ }^{2}$ \\ ${ }^{1}$ Department of Industrial and Systems Engineering, Aoyama Gakuin University, \\ 5-10-1 Fuchinobe, Chuo-ku, Sagamihara, Kanagawa, 252-5258, Japan \\ ${ }^{2}$ Faculty of Business Administration, Kanagawa University, \\ 2946 Tsuchiya, Hiratsuka-shi, Kanagawa, 259-1293, Japan
}

\begin{abstract}
Recently, the manufacturing industry in especially Japan has found it difficult to transfer skills from trained workers to inexperienced workers because the former ages and then retires. This is a particular problem for lathe process, as this operation requires explicit and tacit knowledge, and defining the skills clearly in a manual is difficult. This study aimed to develop a training system for lathe operation by using a simulator; this includes formulas that help define the relationship between the speed of tool feed and cutting sound/shape of chips that were proposed in the preceding study. The present study verified the effectiveness of the proposed training system.
\end{abstract}

Keywords: Lathe operation, Training, Simulator, Cutting sound, Cutting shape.

\section{$1 \quad$ Introduction}

Recently, the manpower, time, and money that the manufacturing industry in Japan can afford are decreasing because the youth are moving away from this industry. Many trained skilled workers had retired around 2007 and it caused their skills disappear. We call it the 2007 problem in Japan. In addition, trained skilled workers are advancing in age and retiring; transferring their skills accumulated over the years is difficult. The Ministry of Economy, Trade and Industry [1] says that $49.4 \%$ of new workers require 2-3 years of training, and $28.1 \%$ require $4-5$ years. In the manufacturing industry, it can take years before a worker becomes sufficiently skilled at his/her work, and even more years are needed by trained workers to acquire advanced skills. In small- and medium-sized manufacturing industries, there is no established system for the transfer of skills, so cost and time have become large problems. The personnel expenses to train a new worker alongside a trained worker are said to double to maintain the production volume and sales at the same level as the previous year [2]. Owing to the lack of technical personnel responsible for the technology infrastructure in terms of both quantity and quality, an education and training method to effectively improve the skill level of a worker in a short period of time is required.

\footnotetext{
* Corresponding author.
} 
This study considered the lathe operation, which was also targeted in the preceding study; this process involves cutting stainless steel into shapes and is performed frequently. The preceding study proposed a method and formulas describing the relationship between the speed of tool feed and cutting sound/shape of chips for the lathe operation based on an experiment with a trained worker [3][4].

The aims of the present study were to develop a training system for lathe operation with a simulator using the formulas proposed in the preceding study, conduct an experiment with the proposed system, and verify the effectiveness of the proposed system.

\section{Devising a Training Method}

The devised training method consists of the following content: explicit and tacit knowledge. Explicit knowledge is transferred by training evaluation, and tacit knowledge is transferred by simulator training [5].

\subsection{Transfer of Explicit Knowledge}

Three aspects of explicit knowledge are described here: sound, handling, and chips.

\subsubsection{Transfer of Sound as Explicit Knowledge}

Sound is transferred as explicit knowledge through a display of the musical scale for the sound frequency [6]. The sound frequency varies depending on the amount of cutting feed; this is converted to a musical scale and displayed. The sound is expressed on the musical scale rather than as a frequency to facilitate better and easier visualization. For example, people can more easily understand the expression "fa \#7 octave" rather than "sound of a $3000 \mathrm{~Hz}$ frequency." A trainee can receive feedback from the musical scale according to the amount of handle feed and difference with the target [7].This item is the original point of this study.

\subsubsection{Transfer of Handling as Explicit Knowledge}

Handling is transferred as explicit knowledge based on the average feed amount, stability of feed rate, difference in feed rate amount, and feed time series average of a trained worker and through a graph of the cumulative amount of feed. The average feed amount is obtained from the simulated feed results [8]. The stability of the feed rate is evaluated according to the standard deviation of five levels: a score of 5 is the best. The differences in the amount of feed rate and feed time series average of a trained worker imply the difference between the absolute value of the time series feed and the objective value of $0.05 \mathrm{~mm} / \mathrm{rev}$ feed. These are evaluated on four levels: best, good, bad, and others. They are also calculated according to the ratio of the previous three items. The total score is calculated on a scale of 100 points using the amount of average feed and stability of the feed rate as base values and the difference in feeding time series feed rate and average of a trained worker as an additional value. The average feed is evaluated on five levels: fast, slightly faster, just right, somewhat slow, and slow in the comments column. The stability is similarly evaluated on five levels: 
very good, good, a little more, bad, and very bad. The graph of the cumulative amount of feed is displayed by pressing a button on the right-hand side of the screen; it compares the results for a trainee and trained worker. The trainee obtains feedback on handling from these items.

\subsubsection{Transfer of Chips as Explicit Knowledge}

Chips are transferred as explicit knowledge based on the diameter and interval of the simulated helix for 3D chip configuration and the thickness and width for the volume of one spiral chip. The 3D simulator shows chips corresponding to the average feed amount being handled. The trainee receives feedback by comparing this figure and the goals.

\subsection{Transfer of Tacit Knowledge}

Three aspects of tacit knowledge are described here: sound, handling, and chips. The transfer of tacit knowledge requires direct communication of the represented sense instead of a numerical representation as for explicit knowledge.

\subsubsection{Transfer of Sound as Tacit Knowledge}

Sound is transferred as tacit knowledge by listening to the simulated sound of cutting. Sound travels from PC speakers (or the equivalent) according to the amount of feed being handled. The trainee judges the quality of the feed by listening to the sound of the pseudo-cutting.

\subsubsection{Transfer of Handling as Tacit Knowledge}

Handling is transferred as tacit knowledge by manipulating handle of the simulator. The amount of handling to feed is acquired by moving a mouse attached to the lathe bench according to the blade movement and handle rotation. The trainee can understand the amount of feed handling by looking at the screen. The black line extends from right to left according to the amount of rotation of the handling and represents the workpiece cutting status. The red line shows the feed amount by a trained worker. The trainee can perform training exercises by comparing his or her feed with the trained worker's feed and level up in rank by adjusting the display after becoming accustomed to a given exercise. The sound frequency of cutting decreases when oil is injected; in the simulation, this is done by left-clicking the mouse. The trainee can acquire the proper amount of feed while actually operating the handle using tacit knowledge.

\subsubsection{Transfer of Chips as Tacit Knowledge}

Chips are transferred as tacit knowledge by looking at the shape. When transferring chips as explicit knowledge, the chip shape is represented by a numerical value. However, actually measuring the diameter and spacing of the chips in actual practice is not desirable because this takes time and effort.

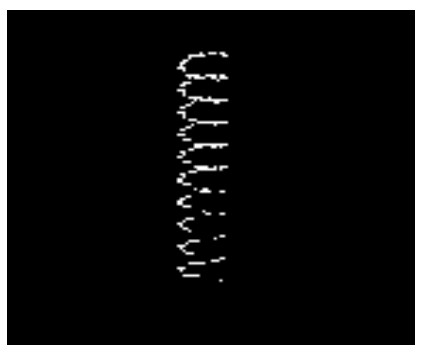

Fig. 1. The shape of the chips 
Ideally, the quality should be judged at a glance (Figure 1). The trainee obtains the chip form as tacit knowledge by visualizing the chip shape according to the amount of feed on the display screen.

\section{The Developed Training System}

The training system consists of the tabletop lathe shown in Figure 2 (Amini No.1100). This tabletop lathe can be used with a household power supply and can create workpieces up to $60 \mathrm{~mm}$ in diameter. This lathe also has processing functions such as cutting an outer diameter and machining chestnut holes as well as a large conventional lathe. It has sufficient functions for training on lathe operations.

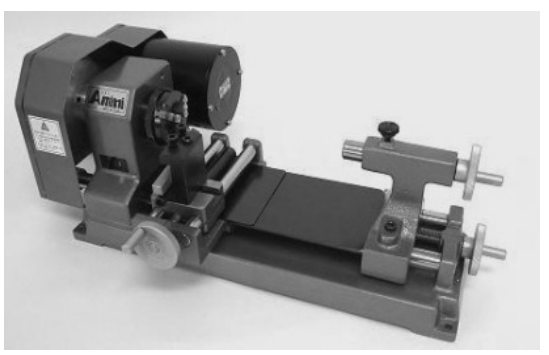

Fig. 2. Tabletop lathe Figure 3 shows the developed training system with this lathe. A mouse is used with this lathe to obtain data on the feeding amount being handled and to simulate lathe operation. The sound of pseudo-cutting can be heard during the simulation, and explicit knowledge on the sound, handling, and chips can be obtained after the simulation. This allows trainees to obtain feedback from the simulated results and learn on their own. The training system is effective and no unnecessary material costs are incurred. On the other hand,

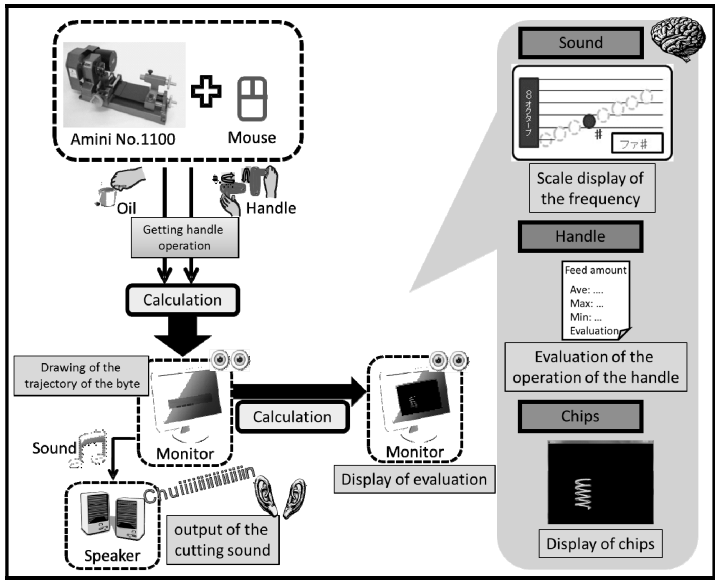

Fig. 3. Overall picture of the training system possible disadvantages include the time and effort needed for development. Therefore, the training system should have a realistic simulation and additional functions not found in practice. In this study, we developed a training system using the free software Eclipse 3.2 and Java.

\section{$4 \quad$ Experiment Design}

Using the training system described in the previous section, the training system was experimentally verified as to whether it effectively improved a trainee's skills by transferring tacit and explicit knowledge on topics such as sound, handling, and chips. Eight university students were selected as subjects and classified into three groups 
based on the preliminary grouping experiment. Two groups of subjects practiced lathe operations with the training system, and the other group practiced without it. The results were then used to compare the degrees of improvement in skills of the three groups.

\subsection{Grouping Experiment}

Eight subjects were classified into three groups based on dexterity so that no differences caused by a subject's individual ability would appear in the subsequent experiment. For the grouping experiment, subjects were asked to make a minicar run on the scoreboard using their hand and guess the number where it would stop in three trials. This experiment measure the subjects' dexterity, coordination, and nerves. These abilities are required for lathe operation.

\subsection{Verification Experiment}

In this experiment, the effectiveness of the developed training system was verified. Two of the three groups (i.e., groups A and B) used the training systems, and group C did not. Group A conducted training using the training system and receiving tips for lathe operation and VTR of feeding behavior from a trained worker. The tips simply summarized the cutting chip sound and how to achieve this or change it with a constant feed rate, how to maintain low resistance during cutting, and how to clean the surface of the workpiece. Group B conducted training using the training system and VTR of the feeding behavior from a trained worker. Group C only saw VTR of the feeding behavior from a trained worker. Viewing VTR is equivalent to the traditional teaching method of apprenticeship; the superiority of the proposed system can be verified through this experiment. Subjects performed lathe operations three times; this involved cutting outlines in stainless steel. They received training during each operation. The termination condition for the training system was more than 85 points for the first time, more than 90 points for the second time, and being successful three times in a row. The upper time limit was 10 min. The training effect for lathe operation was verified based on four criteria: resulting feed amount of the handle, cutting sound frequency, chip thickness, and workpiece surface.

\section{Experiment Result and Discussion}

The eight subjects were put into three groups. The subjects were classified so that the groups would have similar average results. Group A had three subjects with an average of 180.0 points, Group B had two subjects with an average of 180.0 points, and Group $C$ had three subjects with an average of 173.3 points.

The verification experiment was conducted as described above for the three groups. Figure 4 shows a box-whisker plot with the changes in the amount of feed being handled by group A for three lathe operation trials. 
The graph shows the average amount of feed handled by the subjects in the group and the average maximum and minimum values. Group A reached a proper and stable speed for the feed amount being handled after repeated trials. The results for group B were almost the same as those of group A. On the other hand, group $\mathrm{C}$ used a faster speed for the feed amount being handled than the other groups, and the speed was unstable throughout the trials.

The changes in the cutting sound frequency show the average frequency of the subjects in each group and the average maximum and minimum values. For groups $\mathrm{A}$ and $\mathrm{B}$, the lower limit of the frequency decreased in the second trial and increased in the third trial. Confusion over the modified feed amount during training and blank time in the feed amount might have led to the above result in the second trial. For groups $\mathrm{A}$ and $\mathrm{B}$, their skills improved based on the slight decrease in the upper limit of the frequency. For group $\mathrm{C}$, the average frequency decreased, and the upper limit of the frequency was higher than that for the other groups.

Figures 5, 6, and 7 show the changes in the chip thickness. The chip shape is determined by the amount of feed being handled; the chip

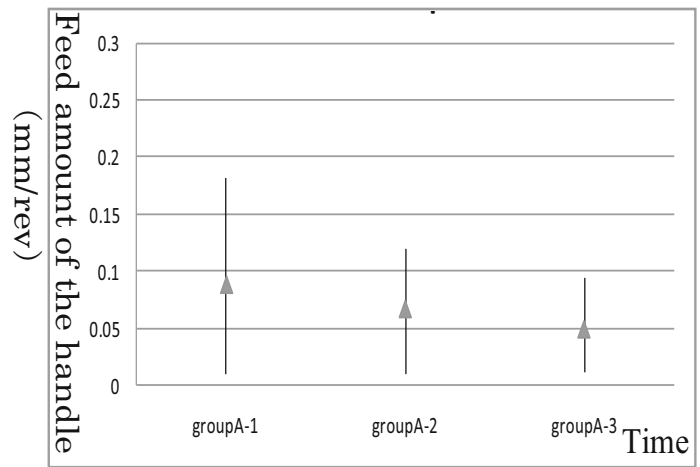

Fig. 4. Amount of feed of the handle of the group A

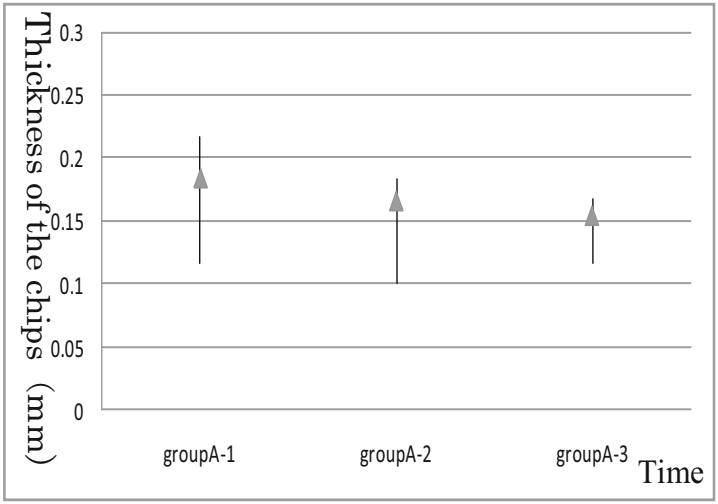

Fig. 5. Changes in the thickness of the chips of group A

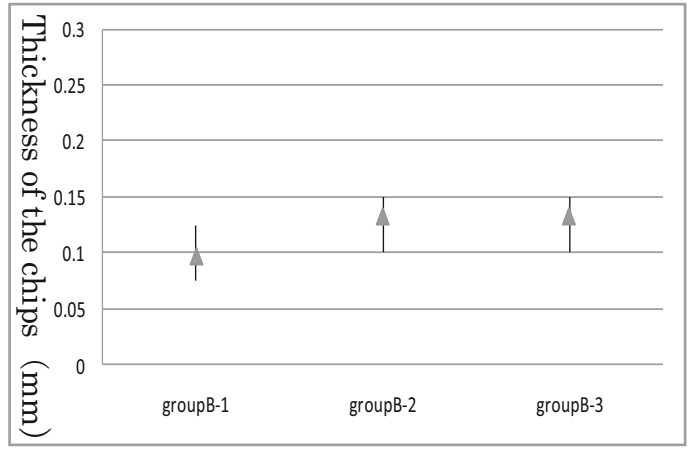

Fig. 6. Changes in the thickness of the chips of group B 
shape thickness is determined independently by the entry spiral and spacing of the front line for the circle width. Only the chip thickness is discussed here. The graphs show the average chip thickness and the average maximum and minimum values of each group. For group A, the average chip thickness decreased. For group B, the value increased. For groups $\mathrm{A}$ and $\mathrm{B}$, the maximum and minimum widths in the third trial were smaller than in the first trial. This means that the training system improved the trainees' skills with regard to chip thickness.

For group $\mathrm{C}$, the average chip thickness was stable, but the value was larger than that of the other two groups, and the maximum and minimum widths showed no tendencies. These graphs were very similar to the transition of the feed amount being handled as shown in Figures 4. Thus, groups A and B, showed improved skill with regard to chip thickness, similar to the improvement in skill for feed

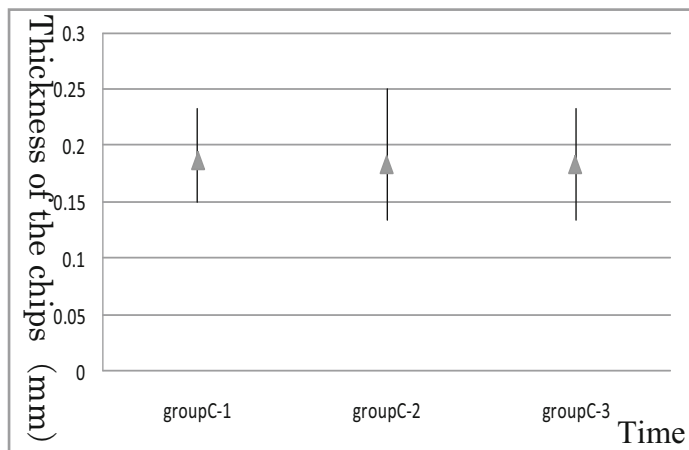

Fig. 7. Changes in the thickness of the chips of group $\mathrm{C}$ amount being handled.

Based on the above, the skills of groups A and B improved, whereas those of group $\mathrm{C}$ did not. The same results were shown on the workpiece surface after machining (i.e., processed surface). Figure 8 shows the surface processed by a subject in group A. The processed surface in the first trial had chatter owing to linear cracks, but the surfaces in the second and third trials were cleaner and had less chatter. The results for group B, were almost the same as those for group A. For group C, whose results are shown in Figure 9, although the chatter of the processed surface in the third trial was thin, the roughness of the surface showed no change. Thus, the skill of groups A and $\mathrm{B}$ with regard to the roughness of the processed surface was improved by the training system.

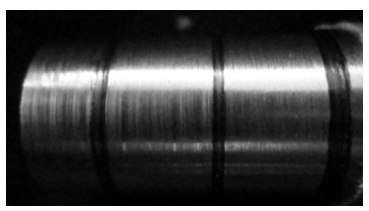

Fig. 8. Group A

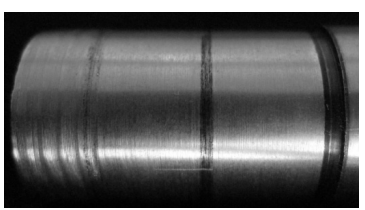

Fig. 9. Group C

\section{Conclusion}

This study proposed a method for transferring explicit and tacit knowledge on lathe operation; a training system was developed with a simulator that uses relationship 
formulas between the speed of the tool feed and cutting sound/shape of chips on lathe operation based on an experiment with a trained worker. The effectiveness of the system was verified through an experiment.

Future work will involve examining the work conditions for lathe operation in detail, improving the training system, and implementing it in actual factories.

\section{References}

[1] Minister of Economy, Trade and Industry of Japan: White Paper in 2007 (2007)

[2] Ryoji, S., Haruo, S., Noburo, E.: Estimation for Proficiency of a Worker by using Cutting Sound. Transactions of the Japan Society of Mechanical Engineers 1997(2), 159-160 (1997)

[3] Takashi, K., Daisuke, D., Michiko, A., Toshiyuki, M.: Basic Study on Relationship between Speed of Tool Feed and Cutting Sound/Shape of Chips on Lathe Work. Journal of the Society of Plant Engineers Japan 24(3), 129-135 (2012)

[4] Hiroshi, N., Atsushi, K., Hiroyuki, S., Hiromasa, Y., Toshiyuki, M., Masaomi, T.: Effect of Rotary Cutting Tool Posture on Machining Performance utilizing Multi-Tasking Lathe. Journal of Advanced Mechanical Design, Systems, and Manufacturing 2(4), 532-539 (2008)

[5] Shoji, T.: New Approach to Handing Down of Implicit Knowledge by Analytic Simulation. The University of Tokyo (2005)

[6] Takeshi, S., Haruo, S., Noburo, E.: Feed Rates Estimation by using Cutting Sound. Transactions of the Japan Society of Mechanical Engineers 41, 171-172 (2006)

[7] Kentaro, N.: Illustration Science - Structure of Sound, Natsumesha (1999)

[8] Tetsuo, F.: Point and Skill for Lathe Process, Nikkan Kogyo Shimbun (2009) 